\title{
Godin, à la rencontre de l'innovation sociale et de l'innovation technologique
}

\section{Gracia Dorel-Ferré}

\section{(2) OpenEdition}

1 Journals

Édition électronique

URL : http://journals.openedition.org/communicationorganisation/2634

DOI : 10.4000/communicationorganisation.2634

ISSN : $1775-3546$

Éditeur

Presses universitaires de Bordeaux

Édition imprimée

Date de publication : 1 mai 2002

ISSN : 1168-5549

Référence électronique

Gracia Dorel-Ferré, "Godin, à la rencontre de l'innovation sociale et de l'innovation technologique », Communication et organisation [En ligne], 21 | 2002, mis en ligne le 27 mars 2012, consulté le 19 avril 2019. URL : http://journals.openedition.org/communicationorganisation/2634 ; DOI : 10.4000/ communicationorganisation.2634

Ce document a été généré automatiquement le 19 avril 2019

(c) Presses universitaires de Bordeaux 


\title{
Godin, à la rencontre de l'innovation sociale et de l'innovation technologique
}

\author{
Gracia Dorel-Ferré
}

1 Godin, c'est, dans le souvenir collectif, la marque de célèbres poêles en fonte. C'est aussi un personnage, qui après avoir connu une grande renommée, était tombé dans un oubli relatif, quand, dans les années 70 , on se reprit d'intérêt pour lui. Il apparaissait alors comme l'industriel qui, issu du monde ouvrier, avait choisi la voie du réformisme et s'était déclaré résolument anti-communiste. Un certain patronat vit à l'époque dans l'expérience de Godin la preuve même que les conflits entre patrons et ouvriers avaient une solution pacifique. Depuis, la chute de l'Union Soviétique et la «fin de l'Histoire » annoncée par certains, a relancé l'intérêt pour cette « utopie pratiquée » dans laquelle on a voulu voir un laboratoire social avant la lettre. Aujourd'hui, à Guise, là où a vécu et travaillé Godin, on met en place un centre d'interprétation de l'œuvre de Godin, autour du thème de l'utopie ${ }^{1}$. C'est peut-être sous estimer l'œuvre de l'industriel novateur, pour qui l'idéal n'était valable que pragmatique et opératoire.

2 On a beaucoup écrit sur Godin, et lui-même a beaucoup écrit de son vivant. Il a déchaîné des passions contradictoires. Son œuvre n'a pas eu d'imitateurs et elle a tenu moins d'un siècle avant d'être totalement abolie. Localement, il a fallu des années pour que l'antagonisme entre les guisards et les « tas de briques » les 
«tas de briques » s'atténue, voire disparaisse. Aujourd'hui, l'hagiographie a triomphé, et on a beaucoup de mal à distinguer de cet amas informatif ce que fut en réalité JeanBaptiste André Godin, entrepreneur audacieux et imaginatif, et concepteur d'une organisation sociale du travail originale. Il a été surtout étudié par les économistes et les sociologues, mais pas par des historiens ${ }^{2}$. En effet, ceux-ci l'ont souvent considéré, en tant qu'industriel, comme un acteur des changements de la vie quotidienne, étude longtemps perçue comme anecdotique. Quant à son action comme patron n'est valorisée que depuis peu, à la faveur des études patronales. Aussi connaît-on mieux le système Godin que les étapes de sa maturation et de sa mise en place ${ }^{3}$. Les personnages de son entourage ont toujours été considérés comme des comparses ou des faire-valoir, en particulier Marie Moret, sa compagne puis son épouse. Celle qui se surnommait volontiers "le porte-plume»" n'a-t-elle pas eu un rôle plus important dans la genèse de l'œuvre sociale de Godin? Elle est responsable de l'image qui est véhiculée, puisque tout ce qui s'est écrit sur Godin, en attendant de sérieuses études d'archives, a été réuni et filtré par elle ${ }^{4}$. Enfin il faudrait distinguer les idées qu'il a exprimées et de celles qu'il a réalisées, dont on fait trop souvent l'amalgame. Ainsi, il faut attendre les années 1880 pour qu'il mette à exécution ses idées sur l'association, et son ouvrage le plus ambitieux, La république du travail, est publié en 1889, par son épouse, après sa mort.

\section{Godin, artisan et entrepreneur}

\section{Un artisan, né dans une famille d'artisans}

Toute la vie de Godin se déroule en Thiérache ${ }^{5}$, cette région verdoyante aux confins de la Champagne, de la Picardie et des Flandres. Voie de passage, région convoitée, déchirée, 
elle se couvre au XVI ${ }^{\mathrm{e}}$ siècle d'églises fortifiées et de temples protestants. Réputée riche d'enfants et pauvre de ressources, elle est traditionnellement métallurgique dans sa partie la plus nordique, mais surtout textile (pour la fabrique de Reims, comme on disait) dans ses bordures méridionales. Godin appartient, par sa naissance, à la première, mais s'installe, comme industriel, dans la seconde. Il y « importe » une activité inhabituelle, ce qui explique en partie les difficultés qu'il rencontrera toujours pour se faire adopter par la majorité de la population.

5 Godin naît en 1817 à Esquéhéries en Thiérache. Il est un fils d'artisan et ne reçoit aucune éducation sinon celle de l'école de son village jusqu'à l'âge de onze ans. Ensuite il est apprenti chez son père et apprend près de lui le métier de serrurier. Mais il cherche à élargir ses horizons, et c'est ainsi qu'on le trouve, en 1834, à Paris, chez son cousin Moret, plus âgé de huit ans et qui aura, toute sa vie, un grand ascendant sur lui. Il a dix-sept ans et plein d'enthousiasme. Moret lui fait rencontrer des milieux saint-simoniens et francsmaçons, qui l'intriguent, mais auxquels il n'adhère pas. Puis il entame son tour de France en 1835. Il passe par Crécy-en-Brie où son oncle a un atelier de serrurerie et va rejoindre son cousin Moret à Bordeaux. De là, il se rend à Toulouse, Marseille, Toulon, Avignon, Lyon. Il vit une expérience inoubliable et fondatrice, qu'il évoquera plus tard dans son premier livre Solutions Sociales publié en $1871^{6}$. Il revient chez lui en 1837. Nous savons de son propre témoignage qu'il avait été frappé durablement par l'injustice de la condition ouvrière, par les bas salaires, les longues journées de travail et surtout la terrible "question du logement », qu'il se promet de contribuer à résoudre, si son industrie lui en donne un jour les moyens.

6 De retour à son pays natal, il reprend le travail familial, se marie en 1840 avec une camarade d'enfance, Esther Lemaire, fille d'artisan comme lui et fonde, grâce à la dot de sa femme, une petite entreprise de chaudronnerie pour laquelle il embauche deux ouvriers. Il a une idée géniale qu'il fait breveter aussitôt et qui lui assurera la fortune : fabriquer des poêles de fonte au lieu de fer qui ne se déforment pas si on les chauffe au charbon. Il faut savoir que les poêles en fer sont fabriqués à la demande, et sont installés de façon provisoire pendant les grands froids. Ils sont chers et donc réservés à une clientèle aisée. Les poêles en fonte sont faits au moule, en série, donc moins chers. Ils sont fabriqués en fonte d'art et constituent un nouveau mobilier, permanent. Cette invention intervient au moment où l'exploitation des mines rend le charbon plus accessible et où les villes s'accroissent, en même temps que sont posées les conditions d'un mode de vie plus confortable. Le succès est immédiat. Dès 1842 , il construit sa propre fonderie dans un hangar voisin. C'est insuffisant. En 1846, il acquiert un terrain près de Guise pour installer un nouvel atelier. Il y emploie trente ouvriers. Quatre ans plus tard, ils sont cent quatre vingt.

7 Son succès, ajouté au fait qu'il adhère au fouriérisme, ouvertement, ne lui facilite pas les relations avec cette petite cité, ancien grenier à sel et sous-juridiction du bailliage de Vermandois qui avait gardé une allure de bourg respectable, et qui eut beaucoup de mal à accepter la formation, à ses portes, d'une ville ouvrière dont les principes d'existence et de fonctionnement étaient si différents. Malgré l'implication de Godin dans la vie municipale puis dans la vie départementale ${ }^{7}$, une profonde mésentente devait s'installer, durablement. 


\section{Un entrepreneur inventif}

8 Au départ, il avait pensé se spécialiser dans les outils aratoires, ce qui montre à quel point ses choix de production ont été précédés d'une vraie réflexion sur ce qui pouvait être innovant, et lucratif. De fait, on a rarement considéré l'œuvre de Godin comme entrepreneur, au prétexte qu'il n'a pas une trajectoire exceptionnelle dans ce XIX ${ }^{\mathrm{e}}$ siècle fertile en capitaines d'industrie. Pourtant, aurait-il pu mener à son terme le Familistère et l'Association sans les revenus considérables qui lui procurait son entreprise?

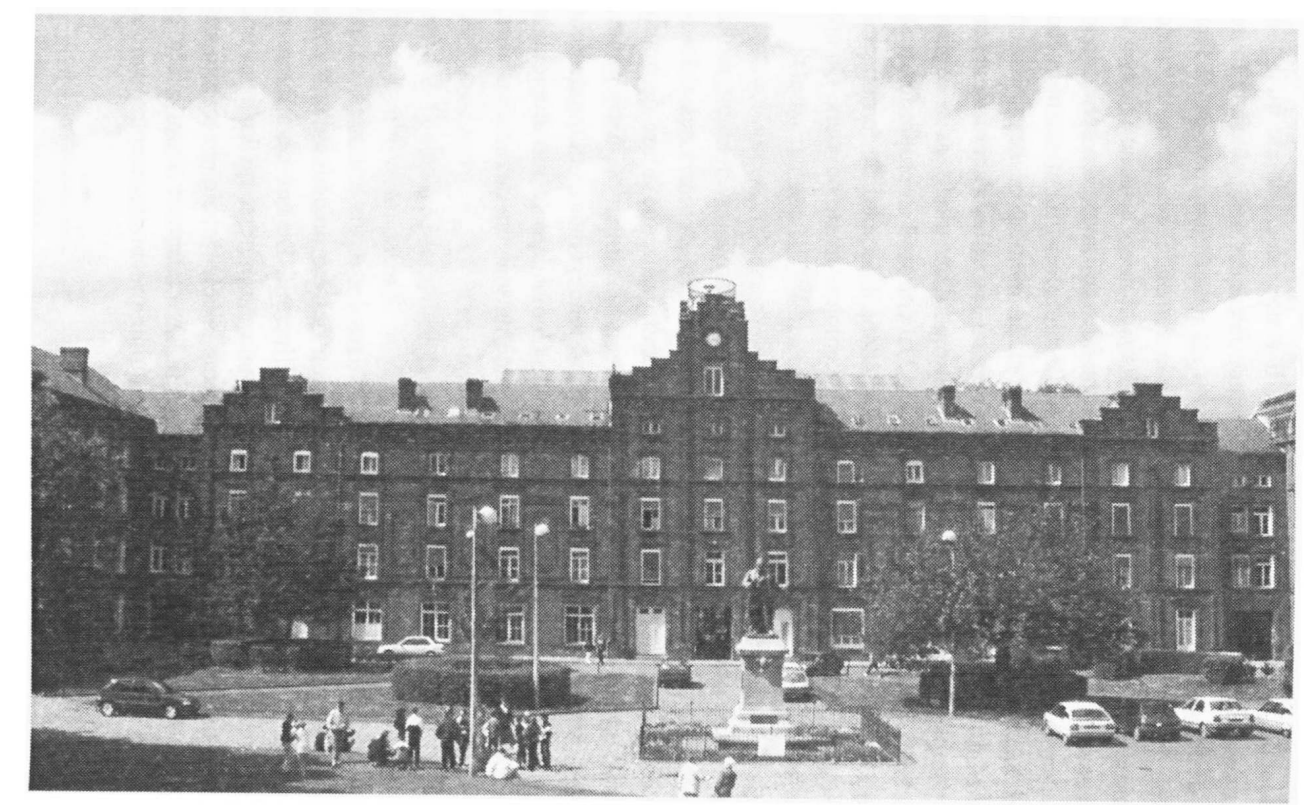

Façade du familistère (1860)

Lui-même pensait que sans les revenus fournis par l'industrie, il n'y avait aucune politique sociale possible.

Aucune étude n'a été dédiée à l'usine ${ }^{8}$, pourtant la première œuvre de Godin, et l'objet de tous ses soins. On ne sait rien des conditions de la production entre 1846 et 1858. L'expansion prise par son industrie conduit Godin à édifier une usine à partir de 1858, dont il poursuit les travaux jusqu'en 1861, et cela bien avant d'entamer la construction du Familistère. La nouvelle usine couvre trois hectares de terrain. On sait que dès 1853. il avait construit une usine à Laeken, près de Bruxelles, où en 1887, il fera édifier une réplique du Familistère, réduite à un seul module. "L'attention du monde industriel écrit-il, était attirée sur moi ».

11 Si l'on sait peu de choses au sujet de la construction de l'usine, du moins la croissance de la population ouvrière est-elle un indicateur éloquent. En 1846, on l'a vu, Godin travaille avec 30 ouvriers; avec 180 en 1850 et 300 en 1857. Ils sont 700 en 1861, lorsque les travaux de l'usine sont achevés. Le nombre ira augmentant puisqu'en 1890, alors que Godin est mort depuis deux ans, on compte 1117 ouvriers. En 1925, la population de l'usine atteint son chiffre le plus élevé : 2000. Notons enfin qu'avec l'usine et avant de construire le Palais Social, il réalise le jardin d'agrément, un élément fort de son projet social. Cette ascension vertigineuse est loin de lui valoir la reconnaissance de ses concitoyens. On ne comprend pas ce transfuge de la classe ouvrière qui se refuse à mener le mode de vie de 
grand bourgeois que sa réussite lui aurait légitimement permis. Mais pour les industriels de son temps, il est un dangereux socialiste. Signe de leur méfiance, malgré tous ses efforts en tant que conseiller général, son usine ne sera jamais reliée au chemin de fer, de son vivant.

12 Sa réussite tient dans sa capacité d'invention. Et son inventivité va de pair avec un sens commercial indéniable doublé d'une grande prudence. Il explique lui-même comment il a évolué dans le domaine de la fabrication et comment il en est venu, poussé par la concurrence à innover sans cesse. Tout reposait sur la nature reposait

Vues partielles de l'extérieur

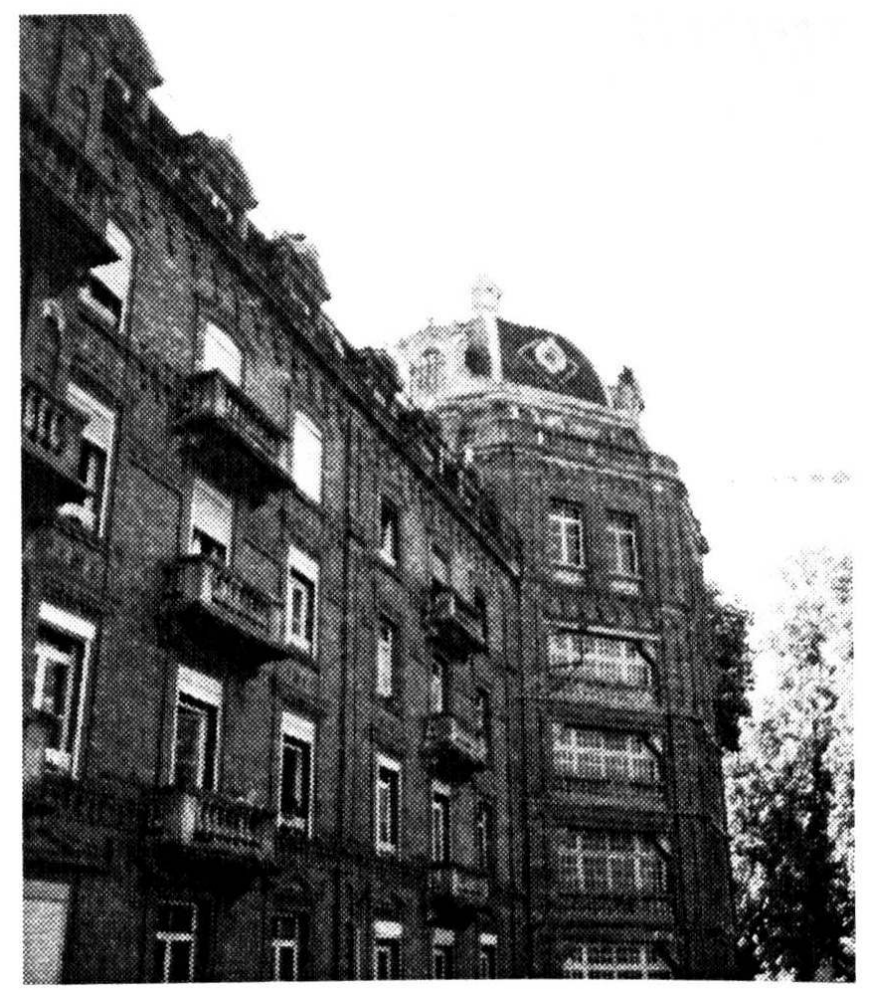




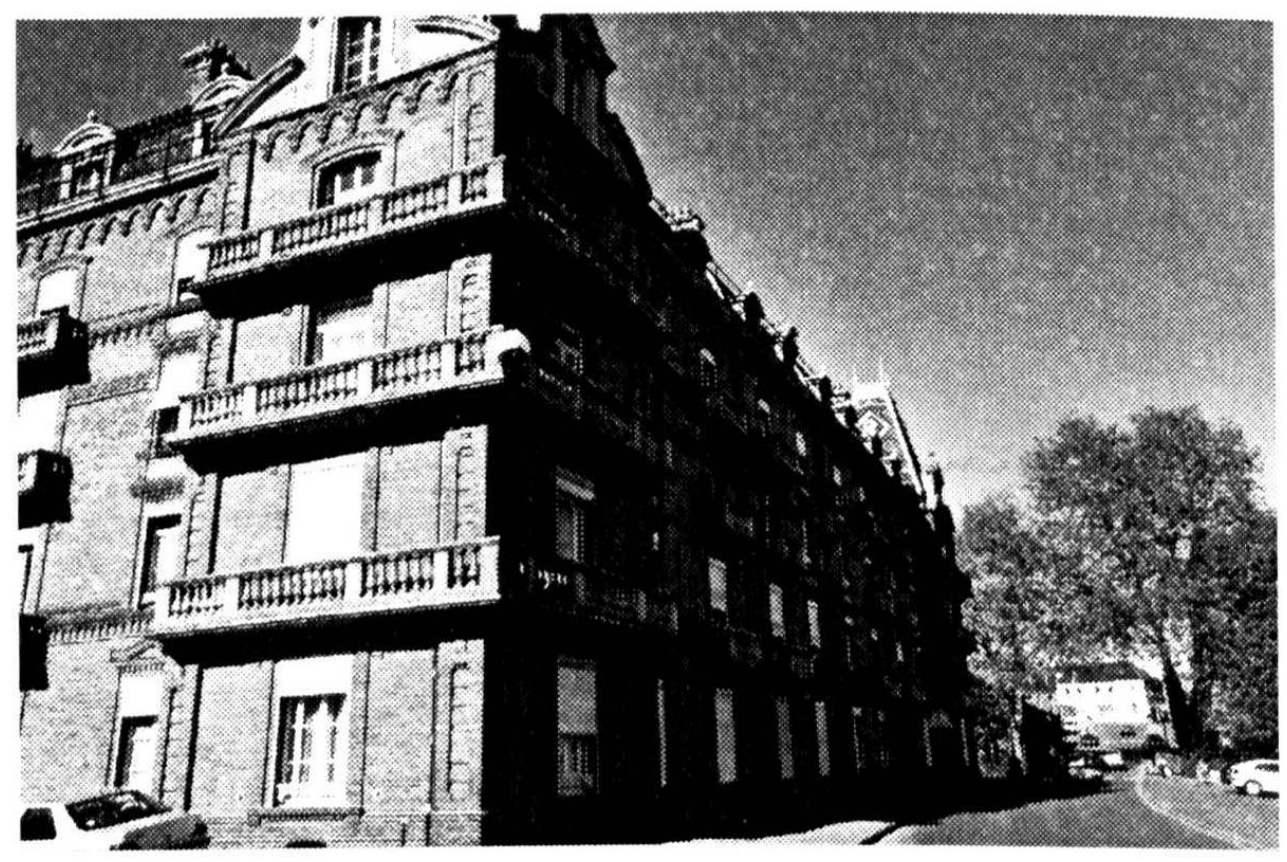

13 sur la nature des brevets qu'il pouvait déposer, qui lui assuraient l'exclusivité de ses modèles et le mettait au moins partiellement à l'abri des contre-façons. Or, les brevets étaient onéreux et de durée limitée. Petit artisan des débuts devenu un industriel dynamique, il déposait sans arrêt les brevets de ses inventions et il inventait sans arrêt. Le poêle, réalisé en fonte moulée, était décliné sous toutes ses formes, depuis la garniture de cheminée de salon jusqu'à la cuisinière, adapté à toutes les catégories sociales, à tous les usages. En 1862, à l'Exposition Universelle de Londres, Godin dépose un brevet pour l'émaillage des poêles de fonte. C'est un succès total. En 1864, il invente un système d'appareils en fonte émaillée inodore, ce qui lui permet de fabriquer à côté des inévitables poêles, des machines à torréfier le café, le cacao, la chicorée, des appareils à chauffer les fers à repasser, les fourneaux des buanderies, les objets de luxe. À partir de 1870, les produits liés à l'hygiène sont de plus en plus nombreux (lavage, repassage, puis lavabos, crachoirs, baignoires, pompes à eau); en 1880, il produit des articles de jardins, gamme qui sera développée par ses successeurs. En 1887, peu avant sa mort, il fera une rapide rétrospective de la fabrication, dans une conférence au personnel en précisant: «La direction ne peut vous maintenir vos salaires que grâce à une incessante création de produits nouveaux donnant lieu à brevets et pat-conséquent à une exploitation exclusive au bénéfice de la société $»$. C'était assez affirmer la relation entre la condition ouvrière exceptionnelle dans la firme et l'innovation technique. En 1860, Godin était certes un patron différent des autres du fait de ses origines populaires auxquelles il restait attaché, de ses idées socialistes qu'il exposait volontiers, mais il était surtout un entrepreneur inventif, avec déjà un quart de siècle d'expérience dans le domaine. Il dérangeait, mais son succès comme entrepreneur l'a certainement protégé des poursuites et des perquisitions que l'on infligeait aux socialistes. Plus encore, son attitude lui valait des conflits domestiques permanents. Esther Lemaire n'acceptait pas que son mari se mette de lui-même au ban de la bonne société, et ne vive pas dans l'environnement habituel des fortunes assises. Contrairement à Godin qui voulait vivre au milieu des ouvriers, Esther habitait une belle demeure bourgeoise dans le goût du temps, à l'extérieur de la ville. 


\section{Une compagne, une partenaire}

14 En 1862, Godin entame son procès en séparation d'avec son épouse qui ne partageait pas ses idées socialistes non plus que la présence, à ses côtés, de la fille du cousin Moret, Marie, une jeune fille de vingt-deux ans, intelligente et cultivée, qui s'était totalement engagée à côté de son prestigieux parent. La venue de Marie conforte Godin dans ses positions philosophiques et le détermine à s'engager plus avant dans l'œuvre sociale qu'il projetait depuis longtemps. En fait, jusqu'à ce moment, Godin avait donné toutes ses forces à son projet industriel, tout en finançant les utopistes fouriéristes dans leur projet de s'installer au Texas. Alors que Godin pensait partir avec eux, et voyant que son fils Emile était encore trop jeune pour prendre des responsabilités dans l'affaire, il avait fait venir à Guise son cousin Moret pour diriger les ateliers pendant son absence. Aussitôt, il avait décelé en Marie, la fille aînée, une intelligence et des dispositions intellectuelles supérieures et l'avait envoyé faire des études à Bruxelles, pendant cinq ans. À son retour, elle était devenue sa secrétaire et sûrement un peu plus. À partir de ce moment, c'est avec elle qu'il va réaliser une œuvre qui dépasse de beaucoup la seule construction du Familistère. Le procès en séparation dure très longtemps : pendant ce temps Godin et sa compagne pratiquent l'union libre au grand scandale de la population locale. Puis Esther Lemaire meurt et Godin peut enfin épouser Marie. Toute sa vie, Marie se situe dans l'ombre de son mari, mais son rôle a été considérable. Godin n'a rien écrit sans elle, au point que l'on peut très logiquement poser la question de savoir qui est l'auteur réel de l'œuvre écrite sous son nom. Marie Moret a elle-même astucieusement brouillé les pistes et établi la « vulgate » à propos de son mari, un ensemble de trois volumes de documents sélectionnés par elle pour constituer la biographie de Godin, sur laquelle, depuis, les historiens se cassent les dents.

\section{Le fouriérisme en action}

\section{Le militant}

15 Il semble que dès son tour de France, Godin a été mis en contact avec les idées qui remuent artisans et ouvriers de ce temps, et que l'on a englobées sous le nom d'utopies sociales. Dès 1835, il aurait été familiarisé avec la pensée saint-simonienne, et au cours de son Tour de France, il aurait été confronté aux idées de Cabet. Il repousse les unes et les autres et s'en est expliqué dans Solutions Sociales. En effet, il devine en Saint-Simon un théoricien des élites; quant à Cabet, il juge son égalitarisme impraticable. Il découvre, semble-t-il, les idées fouriéristes plus tardivement, en 1842, alors qu'il vient de s'installer à Guise, en lisant un article dans le journal Le Guetteur de Saint-Quentin. Il adhère aussitôt à l'Ecole Sociétaire, qu'il soutient financièrement et dont il devient un actif propagandiste.

Godin pouvait-il adopter une autre idéologie que celle-là? Tout ce qui comptait d'hommes politiques influents et d'industriels dynamiques dans le département de l'Aisne se retrouvait dans les cercles animés par les saint-simoniens ou les francs-maçons. Or, il était considéré par tous comme un pestiféré, comme un dangereux partisan des ouvriers. Quant aux idéologies plus radicales, comme le communisme, il y était totalement opposé, en tant qu'ancien artisan attaché à la propriété de ses moyens de production, si modestes soient-ils. 
17 Jusqu'après la révolution de 1848, il ne fait aucun doute que Godin est un fouriériste enthousiaste et s'intéresse même aux projets d'implantation de colonies sociétaires en Afrique ou en Amérique. Il est possible que dès ce moment-là il ait réfléchi aux aspects très pratiques de la construction d'un phalanstère, comme son courrier le laisserait supposer. En dehors de la conception de l'habitat ouvrier, toutefois, les idées que Godin défend, il est vrai des années plus tard, sont assez éloignées de celles du prophète comtois. Rappelons que Fourier a tout basé sur la théorie des passions et sur la conviction que la société a tout intérêt à détourner celles-ci à des fins utiles pour elle, mais pas l'inverse, sauf à produire des frustrés et des malheureux 9 . Godin, conçoit, lui, une véritable religion du travail, dans l'effort et la pénibilité : on est loin de Fourier.

Cependant entre 1846 et 1848, il est sincèrement attaché aux idées de Fourier et c'est comme tel qu'il se présente aux élections de 1848, en républicain convaincu, attaché aux principes de la propriété et de la dignité que confère le travail, fier de sa réussite déjà sensible comme entrepreneur, malgré ses origines modestes. Sans succès. De plus, les Journées de Juin l'éloignent de la masse populaire, et le persuadent que seules les démarches pacifiques et les réformes non violentes sont valables. Cependant, ses engagements lui valent des perquisitions et par prudence, en 1853, il fuit en Belgique, à Laeken, où il installe une usine qui lui servirait de repli le cas échéant.

En 1854, il souscrit au projet de Victor Considérant, d'une "Société de Colonisation Européo-américaine du Texas" dans laquelle il place le tiers de sa fortune. Mais il comprend rapidement qu'une fondation ne peut se faire au mépris d'une organisation économique et dit tout haut son désaccord. En ce sens, il n'est pas un utopiste, s'il l'a jamais été : il est un entrepreneur avant tout. Nous sommes en 1857. Godin. à ce qu'il écrira plus tard, a compris que le rêve américain ne se réalisera pas et décide de mettre en application ses idées, lui-même. Il contacte les architectes Calland et Lenoir, connus pour leurs options fouriéristes et dès 1859 , il entreprend la construction de l'aile gauche du Familistère. Il s'en expliquera plus tard : ce qu'il veut, c'est une construction évolutive, afin de pouvoir l'adapter aux besoins. De fait, il construira le Familistère par module, de 1859 à 1877. Plus tard, il complétera le Palais Social avec les familistères de Landrecies et de Cambrai (1883)

Godin n'avait pas seulement embrassé la cause du socialisme utopique. Il avait aussi adhéré au spiritisme, ce qui ne manque pas d'étonner chez un homme pragmatique et concret. Il s'y adonne dès 1853, après avoir adhéré au magnétisme en 1841. En fait, s'il était très anticlérical, il était aussi profondément religieux et pratiquait une véritable mystique du travail, dans laquelle il trouvait toutes les justifications de ses actes, autre point qui l'éloigné radicalement de Fourier.

21 Mais là comme dans ses idées économiques et philosophiques, Godin cherchait des solutions en harmonie avec ses origines modestes. Seul patron de son temps à avoir ouvertement professé, en France, des opinions jugées subversives, Godin était critiqué très ouvertement et très marginalisé. Sans doute son relatif échec en politique vient-il de ce qu'il n'a jamais voulu se considérer comme un notable, mais comme le guide de ses compagnons. Les chefs d'entreprise de son temps étaient engagés dans la vie politique et mondaine, même s'ils défendaient, comme Menier ${ }^{10}$, des idées progressistes. Ils se retrouvaient dans les cercles de la franc-maçonnerie. Au total, le personnage autant que son œuvre paraissent uniques, un cas particulier de l'Histoire, en même temps qu'une manifestation éclatante de ce que l'industrialisation a pu produire, en un lieu donné, dans 
des conditions particulières, chez une personnalité aux qualités intellectuelles et humaines exceptionnelles.

\section{Une œuvre unique}

Une thèse récente ${ }^{11}$ montre que Godin semble avoir suivi Fourier ou plutôt son disciple Considérant, d'assez près, pour l'édification du Palais Social ${ }^{12}$. Il faut dire que le sujet était à la mode depuis quelques années. Dans sa conception physionomique, le phalanstère avait été popularisé par Eugène Sue, dans les pages célèbres du Juif Errant, à peu près à ces dates-là. En 1848, l'idée avait été lancée, par les révolutionnaires, de la construction d'un phalanstère par arrondissement à Paris. On peut dire que la cité Napoléon en est l'unique réalisation, d'ailleurs inachevée. On y retrouve, comme à Guise, la cour et la galerie de circulation, un mode de construction en hauteur qui n'a rien de spécifiquement fouriériste, mais où l'on fait simplement l'économie de l'escalier. C'est pourquoi on retrouve fréquemment cette disposition dans l'habitat ouvrier contemporain. Cependant, l'immeuble à cour intérieure, du fait des contraintes collectives qu'il suscitait, était considéré comme un modèle d'habitat ouvrier d'inspiration progressiste.

Or dans le même temps, un autre modèle d'habitat ouvrier était mis au point, à Mulhouse : celui de la célèbre cité Dolfuss, où chaque famille disposait d'une maisonnette avec un jardinet. De plus, l'accès à la propriété était rendu possible par un achat de versements fractionnés. Rendre l'ouvrier propriétaire et par conséquent tranquille, était considéré comme une avancée sociale décisive. Godin repousse cette solution. Comme beaucoup d'utopistes de son temps, il pensait que le progrès humain était subordonné aux progrès de l'habitat et de l'architecture. Ce qu'il fallait développer chez l'ouvrier c'était le sens de l'entraide et la collaboration. Entre 1862 et 1870, Godin édifie le Familistère. Le Palais Social, comme son nom l'indique, devait rassembler les critères les plus avancés de l'hygiène et du confort.

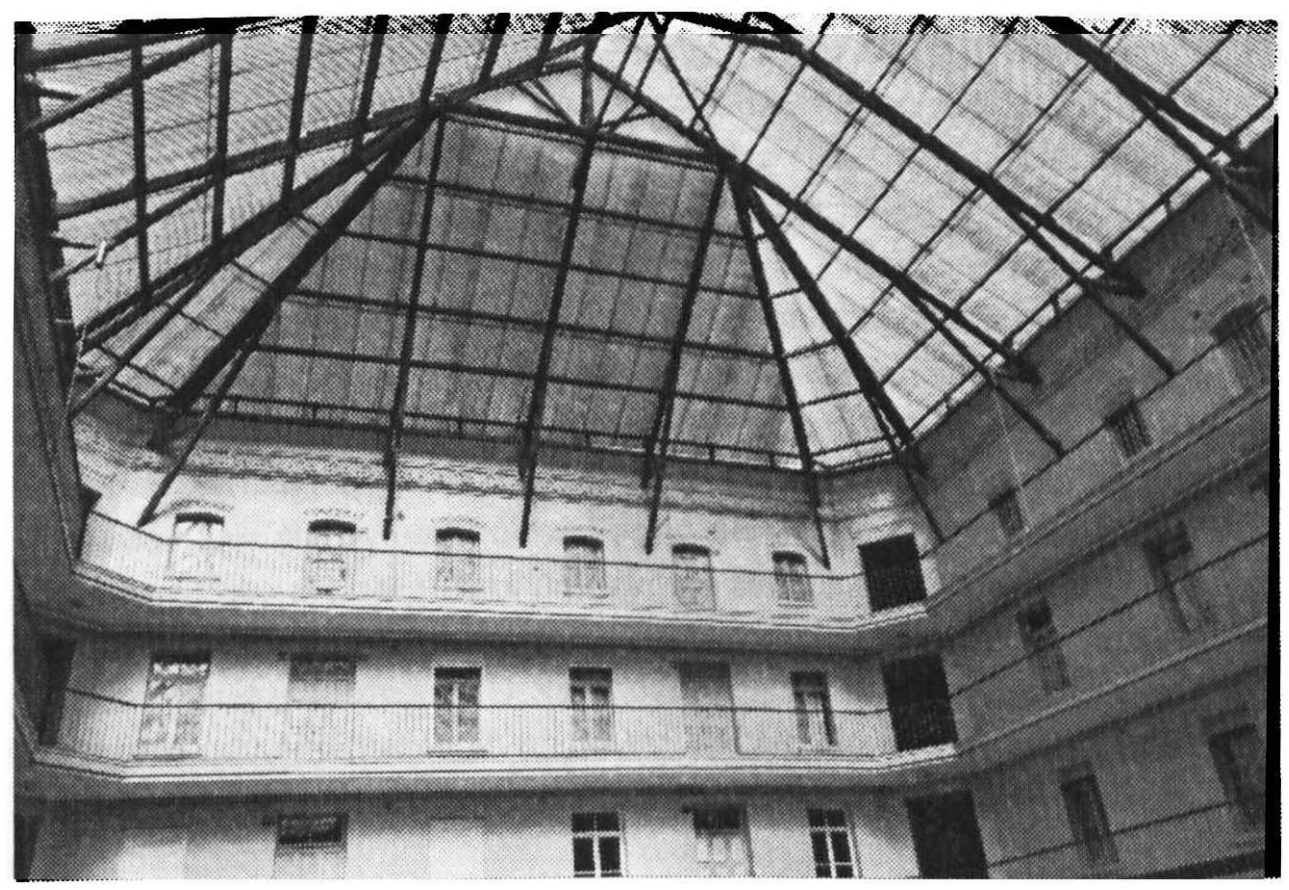


Vue de l' intérieur : la verrière et les appartements.

24 À cette date, le logement ouvrier à initiative patronale comptait déjà d'importantes réalisations ${ }^{13}$. Plus d'un demi-siècle auparavant, Robert Owen avait étonné le monde avec son village ouvrier de New Lanark, où l'accès au logement et aux éléments d'une vie décente allaient de pair avec une éducation par des méthodes actives et une autoévaluation de son propre travail ${ }^{14}$. Par la suite, les colonies ouvrières avaient proliféré dans les régions industrielles, soit sous forme de « corons » ou habitat en rang, soit plus rarement sous forme de blocs maisonnettes d'inspiration mulhousienne. Cependant, ces logements étaient uniformes, exigus et de qualité médiocre. Aussi le thème de l'habitat social est-il récurrent dans tout le XIX siècle. C'est donc par sa conception que le Familistère se distingue des réalisations contemporaines. L'architecture extérieure est inspirée du phalanstère de Fourier, avec des allures de palais classique, d'où le nom que Godin lui a mythe ou réalités, op.cité

donné, de Palais Social ; la conception intérieure, autour de cours vitrées, ramène la vie de chaque module du Familistère vers l'intérieur; les appartements modulables sont exposés à la fois côté rue et côté cour, de façon à être suffisamment éclairés et ventilés ; à chaque étage, des toilettes, des points d'eau, des «trappes à balayures » c'est-à-dire des vide-ordures, une part de cave et une part de grenier, des commerces installés au rez-dechaussée, tout cela assurait aux habitants un niveau de confort que les immeubles parisiens les plus huppés pouvaient à bon droit leur envier. À proximité, l'économat fournissait les aliments mais aussi le charbon, à bon compte. Un peu plus loin, l'eau chaude canalisée depuis l'usine, irriguait un lavoir et une piscine au fond amovible pour que même les enfants puissent apprendre à nager.

Tout ceci n'allait pas sans difficultés : si les premiers compagnons de Godin avaient pu comprendre aisément les buts de leur patron qu'ils côtoyaient directement, il était moins aisé d'obtenir d'une masse nouvellement arrivée des comportements de citadins. Godin avait pris l'habitude de réunir tous les familistériens dans le théâtre pour leur communiquer sa conception de la vie en collectivité. Les propos exigeants du guide étaient accompagnés "d'Avis » placardés sur les murs du Familistère qui dénotent une gestion pour le moins autoritaire du groupe ${ }^{15}$.

À partir de 1864, de nombreux visiteurs viennent au Familistère. Les éloges pleuvent, même de la part d'un pamphlétaire aussi caustique que Frédéric Engels ${ }^{16}$. Il est certain que des années 1870, date de l'achèvement du Palais Social, aux années 1880, où est construit le Familistère Cambrai, il y a eu un ensemble d'avancées tant sur le plan de l'habitat que de la société, remarquables, et pourtant vite déclassées. À la fin du siècle, lorsque Zola vient prendre des notes pour son futur roman, Travail, il a des réflexions plutôt pessimistes devant ce bâtiment où tout, d'après lui, respire le confinement, l'espionnage et la délation ${ }^{17}$. 


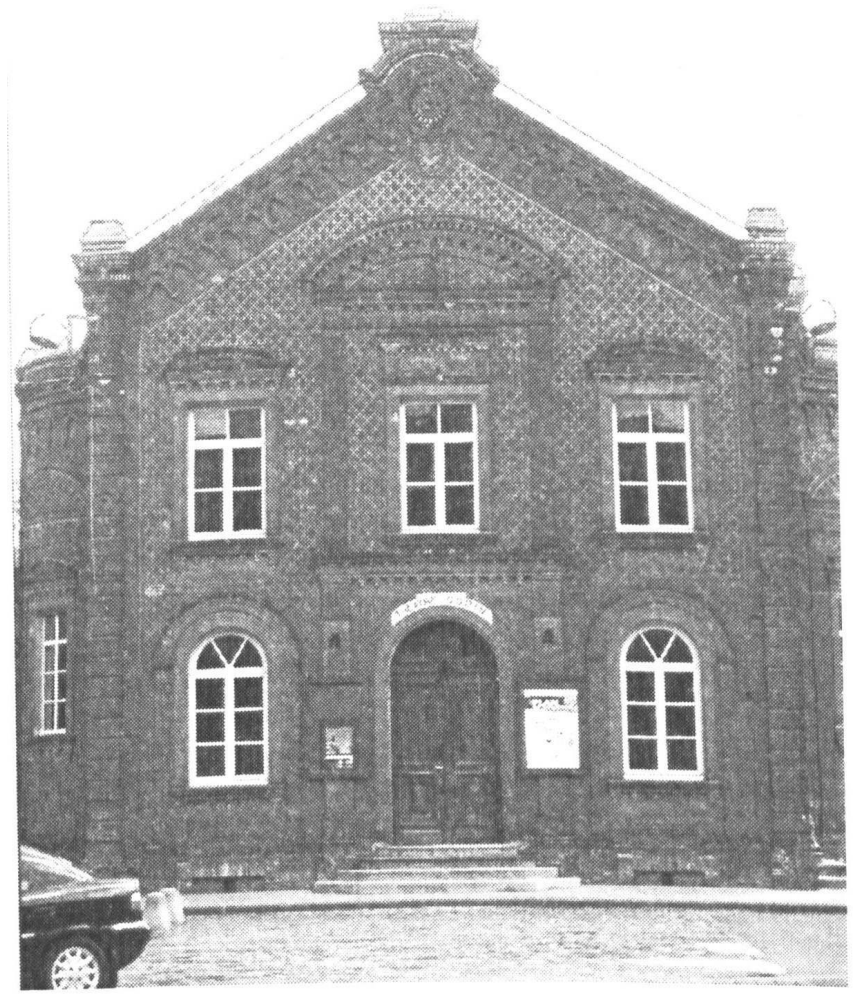

\section{Les équivalents de la richesse}

\section{Une œuvre ambitieuse}

Tout cela a été amplement décrit. Ce qui est unique, c'est le fait que ces avantages aient été accompagnés, à partir des années 1870 , de ce que Godin appelait les équivalents de la richesse. Parmi eux, des avantages sociaux (caisses de maladie, de retraite, etc.) et un système éducatif complet, dans lequel Marie Moret s'engage toute entière.

Depuis la naissance jusqu'à l'âge de travailler, les enfants étaient pris en charge, et passaient de la nourrisserie au bambinat, puis à l'école, et enfin à l'école d'apprentissage. L'éducation était mixte, et les méthodes pédagogiques d'avant-garde. Tous les ans, la fête de l'Enfance réunissait les Familistériens dans la cour centrale du Palais Social et Godin récompensait les meilleurs sujets, au cours d'une célébration au rituel bien établi, plus proche des fêtes des Compagnons que les réjouissances fouriéristes. Quel avenir leur était offert ? L'école professionnelle, sur place, attendait les meilleurs parmi les garçons. Quant aux filles, le trousseau d'entrée à l'Ecole Normale leur était fourni. Notons que Godin, qui militait au sein du conseil général pour que des écoles normales soient construites dans tous les cantons inspire très directement la création de l'Ecole Normale de Laon, œuvre de l'architecte Georges Ermant, mais qui rappelle le Familistère par son architecture, son matériau de construction, et le nom de « Palais de l'Éducation » qui lui est donné.

En dehors de cette perspective, il y avait peu d'embauché féminine sur place, et la plupart des familistériennes étaient femmes au foyer, ce qui ne laisse pas d'étonner quand on se souvient des positions plutôt féministes de Godin. Sauvées de l'ennui par la machine à 
coudre, les dames, au XXe siècle, s'adonnaient à des travaux de couture et d'aiguille, réputés.

31 Si on sépare l'œuvre sociale et le Familistère de l'ensemble de la réflexion de Godin, on constate que bien des aspects de celle-ci ont été pratiquées par ses contemporains: couverture sociale, instruction, modèle petit-bourgeois du mode de vie, logement, etc. Une variante du paternalisme? Menier, pour reprendre cet exemple, avait lui aussi créé une cité ouvrière modèle, de type mulhousien, et avait donné à ses ouvriers un ensemble d'avantages sociaux largement équivalents. Cependant il était un domaine où les patrons de l'époque refusaient de suivre Godin: celui de la répartition des bénéfices et de la participation à la gestion de l'entreprise, à travers une association, même limitée dans ses actions. D'un autre côté, Godin sera suivi difficilement par ses ouvriers qui ne comprendront pas cette curieuse obstination de leur patron. Il faut insister sur ce point : l'association s'est faite contre et malgré eux.

Il y songe dès 1870 puisque l'ouvrage Solutions Sociales en fait état. À cette date, malgré le succès du Familistère et de son industrie, Godin se trouve à la croisée des chemins : il se détourne de la vie politique dans laquelle il n'a pas développé d'affinités. Il a tenté d'instaurer un système d'autogestion dans son entreprise au travers de commissions d'administration élues, mais l'expérience ne dure que deux ans, entre 1870 et 1872 . Il échoue dans un projet de colonie agricole et industrielle dans la Nièvre, en 1875. En 1877, il fait des essais de participation aux bénéfices, qui scandalisent son environnement et consacrent définitivement sa brouille avec son fils, qui jusqu'en 1878, signe la correspondance commerciale. Il estime avoir dû reculer sur bien des plans devant l'ignorance et le manque de dispositions des ouvriers. Il cherche toutefois à pérenniser son œuvre. Il le fait à travers l'Association.

\section{L'Association}

C'est en 1880, huit ans seulement avant sa mort, que Godin dépose les statuts de l'Association du Familistère. Bien connus et étudiés, ils définissent les pouvoirs de l'administrateur et les privilèges de catégories différentes d'associés. L'Association regroupe l'ensemble des dispositions industrielles, économiques et sociales que Godin a prises depuis le début. Mais tout le monde ne fait pas partie de l'Association.

Quatre catégories d'associés sont définies :

- Les participants, dont la conduite et la moralité doivent être irréprochables, sont admis s'ils ont 21 ans et au moins un an de métier. Ils ne sont pas obligatoirement familistériens.

- Les sociétaires sont pris parmi les participants. Ils répondent aux mêmes critères, mais l'ancienneté est de 3 ans et ils habitent le Familistère.

37 - Les associés sont pris parmi les sociétaires. Ils sont l'élite du personnel. Eux aussi doivent être irréprochables, l'ancienneté est plus grande (25 ans d'âge, 5 ans de Familistère, ancienneté de travail 5 ans) ; ils doivent savoir lire et écrire : ils ont une part du fonds social, constituée par l'épargne d'au moins $500 \mathrm{~F}$. Ils sont choisis par cooptation.

38 - Les intéressés rassemblent différents types d'associés qui ont perdu une partie des critères par mise à la retraite par exemple, ou acquisition des parts par héritage, etc.

39 - Les auxiliaires ne font pas partie de l'association mais jouissent d'avantages sociaux (mutuelles). 
Bien sûr, ces distinctions, aux yeux de Godin, reposent sur le mérite. Mais très rapidement dans les faits, les associés sont devenus rapidement inamovibles. Ce sont eux qui peuplent les commissions diverses, qui orientent la politique de l'Association et qui répartissent les bénéfices sous forme de bons d'épargne. L'étude statistique montre qu'ils ont formé un groupe stable, renouvelé par hérédité, soucieux du conservatisme le plus intraitable. En définitive, les statuts ont conduit à la formation d'une élite et une division durable des ouvriers.

\section{Une fin soudaine}

41 Godin disparaît en 1888. Marie Moret, exerce un bref intérim mais refuse à être autre chose que la mémoire de son mari. Une courte gérance est exercée avant que Louis Victor Colin, un brillant ingénieur issu de l'ENSAM de Châlons en Champagne, et fervent disciple de Godin ne prenne la relève, pour 35 ans (1897-1932). Premier d'une courte liste d'épigones, qui ont fait de Godin un dieu de la cité, il ne l'a pas imité dans son audace, ni dans son inventivité. Pour autant, le système mis en place a-t-il, comme le disent certains, remarquablement fonctionné pendant 80 ans environ, ou au contraire a-t-il donné lieu à une véritable lutte de classes interne à l'établissement?

Après la Deuxième Guerre mondiale, le logement social avait beaucoup progressé, la santé et l'éducation étaient désormais une affaire d'État. Beaucoup d'aspects novateurs de l'œuvre de Godin ne l'étaient plus à cette date. De plus, l'innovation technique avait fait place à une confortable routine. L'établissement avait laissé passer la chance du chauffage collectif, pour les immeubles qui se construisaient en nombre. La recherche de bénéfices garantis par et pour les sociétaires avait tari l'imagination, de même que le vieillissement de la population familistérienne, et le dépassement du modèle. La critique communiste, muselée en 1936, avait beau jeu de dénoncer un système qui produisait ses exclus.

Lorsque l'Association dépose son bilan, en 1968, il ne reste plus rien de l'esprit et de l'œuvre de Godin. Le Familistère est vendu, et cet espace qui avait été créé pour être un Palais Social est aujourd'hui le lieu de vie des populations les plus modestes et les moins intégrées de la ville : preuve s'il en est qu'un espace de communication ne peut se réduire à son architecture.

Mais le Familistère est aussi aujourd'hui l'un des patrimoines les plus spectaculaires du nord de la France. L'ensemble, assez bien conservé, est impressionnant. Mis en valeur, il reste le témoignage de l'œuvre d'un homme, et d'une femme, qui ont cherché, dans la tourmente du siècle à concilier l'exploitation de l'homme par l'homme et les valeurs de l'humanisme républicain. Mais l'idéal qu'ils ont représenté n'est plus à l'ordre du jour. Au moment de la construction du Familistère, le plus urgent était de donner à chacun un toit et un univers propre et décent. A la fin du XIX siècle, la question avait notablement évolué. L'innovation sociale se trouvait du côté du concept de la cité - jardin où à côté des œuvres sociales, on mettait en avant le fonctionnement interne de la famille dans un espace différencié et le respect de la vie privée. 


\section{BIBLIOGRAPHIE}

ARNOLD J. « New Lanark, une utopie réalisée » In DOREL-FERRE G. Villages ouvriers utopie ou réalités, op. cité BEECHER J. Fourier, Fayard, 1993, 618 p.

CONSIDERANT V. Description du phalanstère et considérations sociales sur l'architectonique, 1848, réédition GUY Durier, Paris, 1979, 130 p. DELABRE, G. et GAUTIER, J.-M. La regénération de l'utopie socialiste : Godin et le Familistère de Guise, Thèse de doctorat inédite, Paris, 1978 DELABRE, G. et GAUTIER. J.-M. Godin et le familistère de Guise, Vervins. s.d. 330 p.

DELABRE, G. et GAUTIER, J.-M. Vers une république du travail. J.B.A. Godin 1817-1X88, Les éditions de la Villette, 1988, 171 p. DELABRE, G. et GAUTIER, J.-M. J.B.A.Godin et le Familistère de Guise à l'épreuve de l'Histoire, Actes du colloque de Guise du 21 mai 1988, Presses Universitaires de Reims, 1989, $194 \mathrm{p}$.

DEROCHE H. La société festive. Du fouriérisme écrit au fouriérisme pratiqué. Seuil 1975

DOREL-FERRE, G. (dir) Villages ouvriers, mythe ou réalités. Actes du colloque de Guise 16 au 16 octobre 1993, CILAC, 1993, 255 p. ENGELS F. La question du logement, 1872, Editions sociales, 1976, 123 p. GODIN J.B.A. Solutions Sociales, 1871, réédition LA Digitale, 1979, 502 p. LOIRE, M. Le Familistère de Guise, architecture, habitat, vie quotidienne, Mémoire de l'Ecole des Chartes, 1998, inédit.

PAQUOT, T. (Coord.) Le Familistère à Guise. Habiter l'Utopie, Les éditions de la Villette, 1982, 206 p.

PLOUVIER, M. (dir) Sur une des frontières de la France, la Thiérache, Images de patrimoine, 207, l'Inventaire, 2001, $288 \mathrm{p}$.

SMETS M. L'avènement de la cité-jardin en Belgique, Editions Pierre Mardaga, 1977,223 p.

TRELCAT V. L'usine Godin. (1840-1880) une organisation socialiste du travail ? Mémoire de maîtrise inédit, Reims, 1998

VACHER O. «Avis affichés dans un habitat collectif. Du festif au punitif » In Villages ouvriers, utopie ou réalités, op. cité

VALENTIN, M. Noisiel, la chocolaterie Menier, Images du patrimoine, 120, L'Inventaire, 1994

ZOLA E. Carnets d'enquête, édition établie par Henri Mitterand, Plon, 1986, 687 p.

\section{NOTES}

1. II s'agit de la mise en place progressive du projet Utopia,lancé par le Conseil Général de l'Aisne, en partenariat avec la Ville de Guise, la Région et l'Association pour la Fondation Godin.

2. Delabre G. et Gautier J.M. Godin et le Familistère de Guise, une utopie pratiquée en pays picard. Société archéologique de Vervins et de la Thiérache, 1983, 332 p., ouvrage issu d'une thèse en économie, inédite.

Déroche H. La société festive. Du fouriérisme écrit au fouriérisme pratiqué. Seuil, 1975

3. L'important corpus d'archives conservé au Conservatoire des Arts et Métiers à Paris n'a jamais été vraiment exploité, sauf, sur certains points par : 
Vacher O. Avis affichés dans un habitat collectif. Du festif au punitif. Villages ouvriers, utopie ou réalités ? Colloque international au Familistère de Guise, 16-17 octobre 1993, CILAC, 1994.

4. Moret, M. Documents pour une biographie complète de J.B.A.Godin, trois tomes, inédit.

5. Plouvier, M. (dir). Sur une frontières de la France, la Thiérache, Images de patrimoine 207, Inventaire général des monuments et des richesses artistiques de la France, 2001,288 p.

6. Godin, J.B.A. Solutions sociales, 1871, réédition par La Digitale, 1979, 502 p.

7. Il est maire de 1870 à 1874 , conseiller général de 1870 à 1883 , député de 1871 à 1876.

8. Trelcat V. L'usine Godin (1840-1880), une organisation sociale du travail ? Mémoire de maîtrise inédit, 1998.

Il ne donne aucune description de l'espace usinier malgré une étude économique d'un grand intérêt.

9. ${ }^{9}$ Beecher J., Fourier, Fayard, 1993, 618p.

10. Menier est strictement contemporain de Godin. et a eu. lui aussi, des exigences sociales fortes dans sa cité ouvrière. Voir :

Valentin M. Noisiel, lu chocolaterie Menier. Images du Patrimoine 120, L'Inventaire, 1994.

11. Loire M. Le Familistère de Guise, architecture, habitat, vie quotidienne (1858-1968) Mémoire de l'Ecole des Chartes, 1998, inédit.

12. Les idées de Fourier en matière d'habitat social avaient été largement diffusées par Victor Considérant, son disciple. Voir :

Considerant V. Description du phalanstère et considérations sociales sur l'architectonique, 1848; réédition Guy Durier, éditeur, Paris, 1979, $130 \mathrm{p}$.

13. Dorel-Ferré G. Villages ouvriers, mythe ou réalités, op. cité. Une abondante bibliographie est présentée à la suite de chaque contribution. Voir également : Smets M. L'avènement de la cité-jardin en Belgique; histoire de l'habitat social en Belgique de 1830 ci 1930. éditions Pierre Mardaga. 1977, 223 p.

14. Arnold J. « New Lanark, une utopie réalisée ». In Dorel-Ferré G. Villages ouvriers.

15. Vacher O. Du festif au punitif, op. cité

16. Engels F. La question du logement, ensemble d'articles écrits en 1872, Editions Sociales, 1976, 123 p. Celui-ci, d'abord élogieux, finira par voir dans l'expérience de Godin un simple foyer de l'exploitation ouvrière (note de l'édition de 1887).

17. Zola E. Carnets d'enquête, textes établis et présentés par Henri Mitterand d'après les collections de la Bibliothèque Nationale, collection Terre Humaine, Plon, 1986, 687 p.

\section{RÉSUMÉS}

Utopiste pour les uns, réformiste pour les autres, Godin, est avant tout un industriel novateur, pour qui l'idéal n'était valable que pragmatique et opératoire. On connaît l'artisan sensibilisé à la question du logement, mais moins l'industriel inventif, déposant brevet sur brevet pour garantir des revenus sans lesquels il n'aurait pu accomplir son projet social. La communication souligne la fidélité de Godin à ses origines et fait le lien entre innovation et réussite commerciale. Elle souligne les écarts avec la pensée fouriériste qu'on lui attribue en bloc, trop souvent, et l'originalité de ses choix en matière de logement. Elle analyse les raisons de son échec dans la création d'une vraie société participative. L'œuvre monumentale qui subsiste reste une référence universelle. 
Regarded as a utopian by some, as a reformer by others, Godin is primarily a pioneering industrialist, in the eyes of whom the ideal is only acceptable as long as it is practical and operational. The craftsman aware of the housing problem is well known, but less than the inventive industrialist, registering time after time patents to secure income. without which he would have been unable to carry out has social schemes. Social communication highlights Godin's loyalty to his early days and ensures the link between innovation and economic success. It shows the margin between Fourierism which is often wrongly associated with him and his original options in the field of housing. It demonstrates the reasons for his failure in creating a truly participative society. The architectural achievement still standing remains today a universal reference.

\section{INDEX}

Mots-clés : industriel, logement, brevet, fouriériste, société participative

\section{AUTEUR}

\section{GRACIA DOREL-FERRÉ}

Gracia Dorel-Ferré, historienne, thèse sous la direction de Louis Bergeron, soutenue en 1989 sur « Les colonies industrielles en Catalogne, le cas de la Colonia Sedó d'Esparreguera ». S'est spécialisé dans l'histoire des réseaux d'échanges des idées, des personnes et des biens au XIX ${ }^{\mathrm{e}}$ siècle et au début du $\mathrm{XX}^{\mathrm{e}}$ ainsi que dans l'histoire du logement social, dans une perspective comparatiste. Parallèlement, elle travaille à la divulgation et à la mise en valeur du patrimoine industriel en France et en Europe. 